\title{
Linear Algebra Representation of Necker Cubes I: The Crazy Crate
}

\author{
Chris Mortensen \\ Dept of Philosophy \\ The University of Adelaide \\ North Tce, SA 5005, Australia \\ Chris.Mortensen@adelaide.edu.au \\ Steve Leishman \\ Dept of Philosophy \\ The University of Adelaide \\ North Tce, SA 5005, Australia \\ Steve.Leishman@adelaide.edu.au \\ Received by Greg Restall \\ Published March 27, 2009 \\ http://www.philosophy.unimelb.edu.au/ajl/2009 \\ (C) 2009 Chris Mortensen and Steve Leishman
}

\begin{abstract}
We apply linear algebra to the study of the inconsistent figure known as the Crazy Crate. Disambiguation by means of occlusions leads to a class of sixteen such figures: consistent, complete, both and neither. Necessary and sufficient conditions for inconsistency are obtained.
\end{abstract}

\section{INTRODUCTION}

In Mortensen [2006], a logical analysis of the inconsistent image known as the "Crazy Crate" was undertaken, with the aim of demonstrating what the inconsistency amounts to.

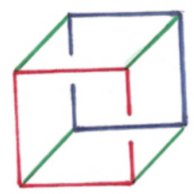

Figure I: The Crazy Crate 
The crazy crate, appearing for example in Escher's masterpiece Belvedere, is a version of the well-known Necker cube, with the important proviso that the standard Necker cube is an ambiguous figure, not inconsistent, whereas the crazy crate disambiguates the figure and produces an inconsistency. In the above paper, disambiguation by means of colours and the convention of occlusion used in the theory of knots enabled sixteen versions of the standard Necker to be identified. Some of these were consistent, some complete, some both and some neither. In particular, two versions of the crazy crate were found, and these were classified as having a certain higher inconsistency, which was termed "global inconsistency". In the present paper, we apply matrix algebra to obtain necessary and sufficient conditions for these various classifications, including global inconsistency. The main project is to find conditions which classify local and global inconsistency both together as kinds of inconsistency. We remain with these sixteen cases, as representing an essential "base case" whose properties need to be set out if the crazy crate is to be described. However, in a sequel these results are extended to the more general case of multiply chained Neckers. For convenience of reference, we reproduce the sixteen cases here. The standard Necker is the top left-hand image, and the two crazy crates are the two bottom right-hand images.
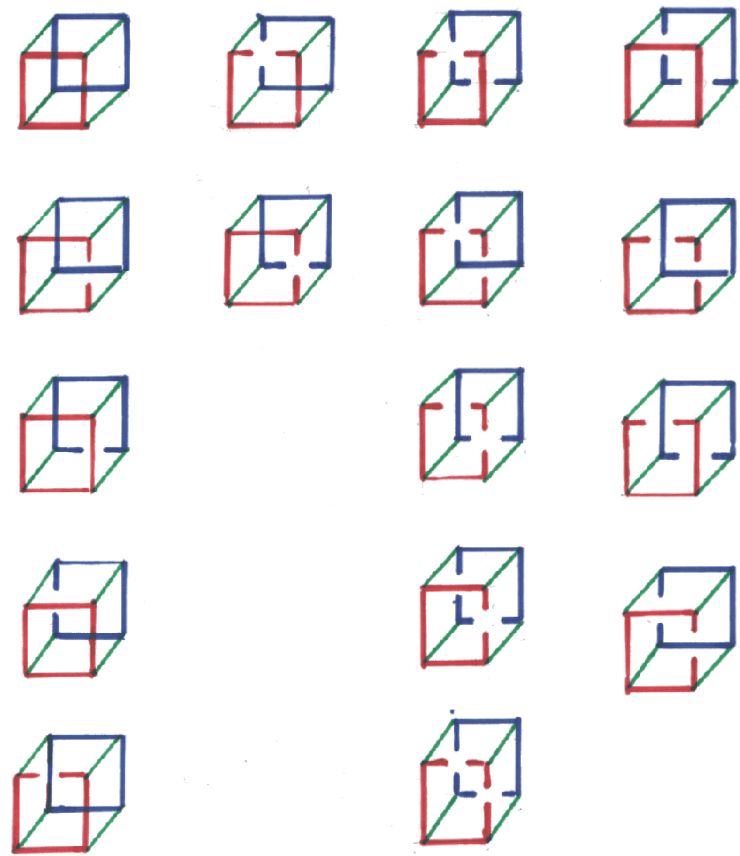

Figure 2: Sixteen Neckers 


\section{PRIMARY AND SECONDARY MATRICES}

In [2006 the faces and their edges were coloured red (R) and blue (B) respectively (with connecting edges coloured green). Each Necker diagram above has two places where red crosses over (occludes) blue or vice versa. These are termed crossings, $\mathrm{C}_{1}$ (left hand crossing) and $\mathrm{C}_{2}$ (right hand crossing). The Neckers differ from one another in just two places, hence we ignore all other data on the images as constant and therefore irrelevant to their differences. The presence or absence of $R$ and $B$ at the crossings $C_{1}$ and $C_{1}$ completely distinguishes all sixteen. Thus for each cube there is a pair of simultaneous linear equations:

$$
\begin{aligned}
& a \cdot R+b \cdot B=C_{1} \\
& \text { c. } R+\text { d.B }=C_{2}
\end{aligned}
$$

where each of $a, b, c, d$ are in $\{0,1\}$. For example, " $1 . R+O B=C_{1}$ " can be read " 1 of red plus 0 of blue make up crossing $C_{1}$ " In turn, the simultaneous linear equations can be written in the usual fashion as a matrix multiplication over the field $Z_{2}$, that is $Z \bmod 2$ :

$$
\left[\begin{array}{ll}
\mathrm{a} & \mathrm{b} \\
\mathrm{c} & \mathrm{d}
\end{array}\right] \cdot\left[\begin{array}{l}
\mathrm{R} \\
\mathrm{B}
\end{array}\right]=\left[\begin{array}{l}
\mathrm{C}_{1} \\
\mathrm{C}_{2}
\end{array}\right]
$$

We call this the PRIMARY EQUAT ION of the cube, and the left hand $2 \times 2$ matrix the PRIMARY MATRIX. If $M$ is a primary matrix, we also denote it as $M_{p}$.Given a matrix designated as a primary matrix , the Necker figure can obviously be recovered.

In [2006], the two crazy crates are described as locally consistent but globally inconsistent, as exemplified by a failure of (consistent) face building. The status of faces in these figures can also be represented by linear equations over $Z_{2}$. We write:

$$
\begin{aligned}
& a^{\prime} C_{1}+b^{\prime} C_{2}=R F \\
& c^{\prime} C_{1}+d^{\prime} C_{2}=B F
\end{aligned}
$$

where as before the (primed) coefficients are from $\{0,1\}$. For example, "1. $C_{1}+$ $0 . C_{2}=R F$ " can be read as "The red face is made up by having red at $C_{1}$ and not having red at $\mathrm{C}_{2}$ ” As before, these equations can be written in matrix form:

$$
\left[\begin{array}{ll}
\mathrm{a}^{\prime} & \mathrm{b}^{\prime} \\
\mathrm{c}^{\prime} & \mathrm{d}^{\prime}
\end{array}\right] \cdot\left[\begin{array}{l}
\mathrm{C}_{1} \\
\mathrm{C}_{2}
\end{array}\right]=\left[\begin{array}{l}
\mathrm{RF} \\
\mathrm{BF}
\end{array}\right]
$$

We call this the SECONDARY EQUATION of the corresponding Necker, and the left hand $2 \times 2$ matrix its SECONDARY MATRIX, denoted by $M_{s}$.

In linear algebra, the TRANSPOSE of a matrix $M$, written $M^{\mathrm{T}}$, is the matrix made up by exchanging rows for columns and vice versa. That is, if $a_{i j}$ is is the element in $M$ in the $i$-th row and $j$-th column, and $a_{i j}^{\prime}$ is the element of 
$M^{T}$ in the $i$-th row and $j$-th column, then $a_{i j}^{\prime}=a_{j i} \cdot M^{T}$ is the reflection of $M$ across the main diagonal. Evidently, the operation $\mathrm{T}$ is an involution, that is $\mathrm{M}^{\mathrm{TT}}=\mathrm{M}$.

THEOREM I Let $\mathrm{N}$ be any Necker cube with primary matrix $\mathrm{M}_{\mathrm{p}}$ and secondary matrix $M_{s}$ Then $M_{s}=M_{p}^{T}$.

Proof: From the simultaneous equations, we can deduce that the red face is made up of contributions of $a . R$ at $C_{1}$ and c.R at $C_{2}$. Thus, a. $C_{1}+c . C_{2}=$ RF. The other case is similar, giving b. $C_{1}+$ d. $C_{2}=B F$. Assembling the two equations into the secondary matrix gives the result. (Alternatively, one may prove this result by enumeration of cases and inspection thereof.)

It is recalled from 2006 that a Necker cube was classified as LOCALLY INCONSISTENT just in case it has both colours, $R$ and $B$, at one or both crossings. It is apparent that this corresponds to having all $1 \mathrm{~s}$ in either row of its primary matrix. The special case where both rows are all $1 \mathrm{~s}$ is the Necker cube familiar from psychology texts. We call its matrix the TRIVIAL matrix, corresponding to the trivial theory (in which every statement holds). The locally inconsistent Neckers are the left hand column of the diagram; the trivial Necker being the topmost. Thus, a Necker is locally consistent just in case no row of $M_{p}$ is all $1 \mathrm{~s}$.

A Necker was further classified as INCOMPLETE if one or both crossings have no colours present. It is apparent that this corresponds to having all $0 \mathrm{~s}$ in either or both rows of $M_{p}$. The case where both rows are all Os corresponds to the theory having no (non-logical) statements holding. The incomplete Neckers are the two middle columns of the diagram. Thus, a Necker is complete if no row of $M_{p}$ is all $0 s$.

Of the four locally consistent and complete Neckers (the right hand column), the bottom two, the crazy crates, are inconsistent in a further global sense. As noted before, this was explained as a failure of consistent face building. A consistent face is built just in case its colour is present at both crossings, or absent at both crossings, corresponding to being unambiguously the front face, or unambiguously the back face. It is apparent, therefore, that a face is built just in case its corresponding row in the secondary matrix is all 1s, or all Os. So, for these four locally consistent and complete Neckers, to be globally consistent is to have both faces consistently; that is no row on $M_{s}$ is all 1s and no row is all 0s. Since $M_{s}=M_{p}^{T}$, it follows that no column on $M_{p}$ is all 1s and no column is all Os. These are the top two Neckers of the fourth column.

On the other hand, since the two crazy crates also have no rows all 1s and no row is all Os, we can isolate these two globally inconsistent Neckers as having respectively one of the two primary matrices:

$$
\left[\begin{array}{ll}
1 & 0 \\
0 & 1
\end{array}\right] \text { and }\left[\begin{array}{ll}
0 & 1 \\
1 & 0
\end{array}\right]
$$


These are the crazy crates. The first of these is the DIAGONAL matrix whose leading diagonal is all $1 \mathrm{~s}$ and there are just Os elsewhere, and we name it ID here. The second is the ANTIDIAGONAL matrix whose antidiagonal is all $1 \mathrm{~s}$ and there are just Os elsewhere, and we name it ANTI-ID here. We observe, in passing, that in each case, $M=M^{T}$, and that, in general, $M_{p}=M_{s}$ iff $M=M^{\mathrm{T}}$.

We utilise this information in what follows.

\section{DETERMINANTS}

We proceed to several "tests" for inconsistency and incompleteness. We are expecially seeking matrix-algebraic conditions which group together the two kinds of inconsistency as being similar. The first obvious test to apply is the nature of the determinant of the matrix. Unfortunately, as we see, while this yields some information, it is a blunt instrument.

The DETERMINANT of the primary matrix given at the beginning of the last section is the sum $(a d-b c)$, that is the product of main diagonal less the product of the antidiagonal. The determinant of a matrix $M$ is written $\operatorname{Det}(M)$. (In $n \times n$ matrices the definition of the determinant is more complicated. The reader also is reminded that the arithmetic being used is that of $Z_{2}$.)

THEOREM 2 If $\operatorname{Det}(M p) \neq 0$, then $M_{p}$ is inconsistent.

Proof: If $a d-b c \neq 0$, then we have two cases. Case (a): $a=d=1$ and one or both of $b, c=0$. If one of $b, c=1$ then we have three $1 \mathrm{~s}$ in the matrix, so that one row is all $1 \mathrm{~s}$, so that the matrix is inconsistent. If both $b, c=0$, we have the diagonal matrix which was identified above as one of the two globally inconsistent Neckers. Case (b): $b=c=1$ and one or both of $a, d=0$. If one of $a, d=1$ then we have three $1 \mathrm{~s}$ in the matrix which is inconsistent. If both $a, d=0$, we have the antidiagonal matrix which was identified above as the other of the two globally inconsistent Neckers.

COMMENT We note that the converse fails in three cases: the trivial matrix and the two inconsistent and incomplete matrices (a row of $1 \mathrm{~s}$ and a row of $0 \mathrm{~s}$ in $M_{p}$ ). Thus it would seem that the determinant is not such a useful instrument in identifying consistency and inconsistency.

\section{NULLITY}

A more precise test is given by the concept of nullity. From linear algebra, we have the notion of the null space. The NULL SPACE of an $n \times n$ matrix $M$ is the space of (consistent) solutions of the matrix equation $M x=\underset{\sim}{0}$, where the unknown $x$ is an $n$-ary column vector of variables, and $\underset{\sim}{0}$ is the $n-$ ary column vector which is all zeros. The NULLITY of the matrix is the dimension of the null space. Note that the matrix equation $M x=\underset{\sim}{0}$ always has at least one 
solution, namely all the variables equal to zero. If this is the only solution, it is termed the zero-dimensional solution.

THEOREM 3 Let $\mathrm{M}_{\mathrm{p}}$ be non-trivial. Then $\mathrm{M}_{\mathrm{p}}$ is complete iff the nullity of $\mathrm{M}_{\mathrm{p}}$ is zero.

Proof: Left to Right: If $M_{p}$ is complete then no row is all Os. If $M_{p}$ is also non-trivial, then not all entries are 1s. So the number of $1 \mathrm{~s}$ is 2 or 3 . If any row is all $1 \mathrm{~s}$, then only the solution $(R, B)=(0,0)$ is possible. If no row is all $1 \mathrm{~s}$ then $M_{p}$ is either the diagonal matrix or the antidiagonal matrix. In each case the nullity is zero since the occurrences of 1 force that variable to be zero. Right to Left: If $M_{p}$ is incomplete then one of both rows are all Os. Any row of all Os does not disturb the set of solutions, since everything is a solution to it. Thus the space of solutions is that of the other row. But this must have at least one other solution: if the row is both $1 \mathrm{~s}$ then $[1,1]$ is a solution, if the row has one 1 then a zero in that place and anything in the other place suffices for a solution, and if the row is both zeros then anything is a solution.

COMMENT From the theorem, a non-zero nullity indicates that there are other ways to satisfy the matrix equation. The nullity can thus be thought of as a measure of the collection of ways in which $R$ and $B$ come together to make an assignment to the crossings $C_{1}$ and $C_{2}$. A non-zero nullity indicates that there are more ways of doing this than simply having no colours anywhere. This in turn is a mark of incompleteness: an incomplete crossing can be completed in more than one way, which indicates more than one solution for $[R, B]$.

However, note too the exception, when $M_{p}$ is trivial. This is complete and inconsistent. However, $[R, B]=[1,1]$ is also a solution of the nullity equation, since in $Z_{2}, 1+1=0$. So the nullity of $M_{p}$ is non-zero. However, its converse (incompleteness implies non-zero nullity) holds irrespective of triviality.

\section{THE STRUCTURE OF THE NULL SPACE.}

The above suggestive result invites a related test for inconsistency and incompleteness, namely to look at the structure of the null space of the primary and secondary matrices. In an improvement over the previous sections, the conditions obtained are general.

THEOREM $4 A$ Necker is inconsistent iff the null space of its primary matrix satisfies $\mathrm{R}=\mathrm{B}$.

Left to Right: If a Necker is inconsistent then we have either that at least one row is $[1,1]$, or is the diagonal or the antidiagonal. If any row is $[1,1]$ then the only way this can compute to zero is if $R=B=0$ or $R=B=1$. Either way, $R=B$. For the diagonal matrix we have one row $1 . R+0 . B=0$, which implies $R=0$. The other row is $0 . R+1 \cdot B=0$, which implies $B=0$. Hence $R=B$. The calculation for the antidiagonal matrix is the same. Right to Left: Any zero entry in the primary matrix, and thus the row $[0,0]$, does not disturb the set of solutions The row $[0,1]$ implies that $B=0$ and $R$ is any, so that $R=1$ is possible, 
so that $R \neq B$ is possible. The calculation for the row $[1,0]$ is the same. Hence matrices with three zeros do not satisfy $R=B$. Matrices with three or four ones are all inconsistent. This leaves six. Two of these, the diagonal and the antidiagonal, are globally inconsistent. Two more, having a row of $1 \mathrm{~s}$ and a row of Os, are locally inconsistent. The final two, where the two rows are identical, force one colour to be 0 while the other colour can be any, so $R \neq B$ is possible.

COMment The test asks which combinations of $R$ and $B$ make for each crossing to be zero. The inconsistent Neckers are just those for which the primary equation requires $R=B=0$ or $R=B=1$. This is perhaps not surprising in that $R=B=0$ is always a solution, and otherwise only $1.1+1.1=0$ gives an inconsistent row.

THEOREM 5 A Necker is complete iff the null space of its secondary matrix satisfies $\mathrm{C}_{1}=\mathrm{C}_{2}$.

Proof: Left to Right: A Necker is complete iff no row of its primary matrix is all zeros. Hence iff no column of its secondary matrix is all zeros. Such matrices have at least two ones in them. Matrices with exactly two ones and no column all zeros are (a) the diagonal, (b) the antidiagonal, or (c) one row all ones and the other all zeros. Case (a) gives 1. $C_{1}+0 . C_{2}=0$ which forces $C_{1}=0$; which combined with 0. $C_{1}+1 . C_{2}=0$ also forces $C_{2}=0$; whence $C_{1}=C_{2}$. Case (b) is the same. Case (c): the row of all ones forces $C_{1}=C_{2}=1$ or 0 . Otherwise, matrices with at least three ones in them force $C_{1}=C_{2}$, because as before the row 1. $C_{1}+1 . C_{2}=0$ requires $C_{1}=C_{2}=0$ or 1 . Right to Left: Conversely, if a Necker is incomplete, then there is some column of the secondary matrix which is all zeros. Without loss of generality, we may assume it is the left column. Columns of the secondary matrix correspond to crossings. So the value of that crossing can be either 0 or 1 and the secondary equation is still satisfied. If the other column is also all zeros then the matrix is all zeros and all solutions are possible so that $C_{1} \neq C_{2}$ is possible. If the other column has at least one $1 \mathrm{in}$ it, then that column is forced to be 0 , since $0 . C_{1}+1 . C_{2}=0$ implies $C_{2}=0$. Hence $C_{1}=1$ and $C_{2}=0$ is a solution, and thus $C_{1} \neq C_{2}$ is again possible.

comment This is a little surprising, because one would think that it is the nature of the primary equation that determines consistency/inconsistency and completeness/incompleteness. Problem: how to turn this into a condition on the null space of the primary matrix? 


\section{THE UNIT EQUATION}

The results of the previous section suggest another test for inconsistency and incompleteness, namely to look at the UNIT EQUATION of (the secondary matrix of) the Necker. This is defined as the equation $M_{s} x=\underset{\sim}{1}$, where $x$ is a column vector of variables and $\underset{\sim}{1}$ is the unit column vector having all ones.

THEOREM 6 A Necker is inconsistent iff its unit equation has solutions.

Proof: Left to Right: If a Necker is inconsistent then either (a) some row of its primary matrix is all $1 \mathrm{~s}$, or the primary matrix is (b) the diagonal or (c) the antidiagonal matrix. If (a) then some column of its secondary matrix is all $1 \mathrm{~s}$. wLOG, let it be the first column, that for $C_{1}$. Then $C_{1}=1$ and $C_{2}=0$ is a solution, since the equations are of the form $1 . C_{1}+$ any. $C_{2}=1$. The argument is similar if it is the second column. If (b) then the equations are 1. $C_{1}+0 . C_{2}=1$ and 0. $C_{1}+1 . C_{2}=1$, which has $C_{1}=C_{2}=1$ as a solution. Case (c) is similar. Right to Left: If a Necker is consistent then no row of $M_{p}$ is all $1 \mathrm{~s}$, so no column of $M_{s}$ is all $1 \mathrm{~s}$. Suppose that it has one 1 in it. If the other column is all zeros, then we have a situation like 1. $C_{1}+0 . C_{2}=1$ and $0 . C_{1}+0 . C_{2}=1$, which have no solutions. Similarly, if $M_{s}$ is all zeros then the unit equation has no solutions, since each row would have to satisfy $0+0=1$. The final two cases are where the Necker is consistent and complete, but in this case the equations look like 1. $C_{1}+1 . C_{2}=1$ and $0 . C_{1}+0 . C_{2}=1$, which have no solutions.

THEOREM 7 A Necker is complete iff the unit equation of its primary matrix has solutions.

Proof: Left to Right: If a Necker is complete then no row of its primary matrix is all zeros. Thus $M_{p}$ has 2 or more ones. If it has 4 ones then it is easy to see that either $R=0, B=1$ or $R=1, B=0$ are both solutions. If it has 3 ones then the row with the zero in it, eg. $1 . R+0 . B=1$, forces $R=1, B=0$, which in turn satisfies the second row $1 . R+1 . B=1$. If it has two ones, then there are three cases: (a) two consistent and complete, (b) the diagonal, and (c) the antidiagonal. In case (a) we have eg. $1 . R+0 . B=1$ and $1 . R+0 . B=1$ which is satisfied by $R=1, B=$ any. In case (b) we have $1 . R+0 . B=1$ and $0 . R+1 . B=1$ which has the solution $R=B=1$. Case (c), the antidiagonal, is the same. Right to Left: If a Necker is incomplete than at least one row of $M_{p}$ is all zeros. This makes it impossible to satisfy the primary equation since for that row $0 . R+0 . B=1$, so there are no solutions.

\section{CONCLUSION}

Theorem 6 in particular supplies the desired assimilation of local and global inconsistency. The unit space of the secondary matrix is the test of inconsistency of whatever kind. This justifies the use of the secondary equation, 
defined as representing ways of face building. To be inconsistent, both faces must have a value of 1 in the secondary equation, whereas to be consistent each face must have a value zero, corresponding to a face being consistently behind the other, or consistently in front. The contents of the unit space catalogue the ways that inconsistent Neckers are generated. Additionally, the final Theorem shows that the unit space of the primary matrix catalogues the ways that a Necker may be complete.

It is pleasing that $2 \times 2$ matrices over the simplest case of a field, namely $Z_{2}$, are adequate to describe these cases. Indeed, the proofs in each case could be replaced simply by inspection of cases, though the proofs given above carry some theoretical illuminations. In a sequel, it is described what is preserved and what is lost in moving to the more general case of $n$ Neckers.

\section{REFERENCES}

[2006] MORTEnsen, Chris, (2006) "An Analysis of Inconsistent and Incomplete Necker Cubes", Australasian fournal of Logic, 4, 216-225. 
The Australasian fournal of Logic (ISSN I448-5052) disseminates articles that significantly advance the study of logic, in its mathematical, philosophical or computational guises. The scope of the journal includes all areas of logic, both pure and applied to topics in philosophy, mathematics, computation, linguistics and the other sciences.

Articles appearing in the journal have been carefully and critically refereed under the responsibility of members of the Editorial Board. Only papers judged to be both significant and excellent are accepted for publication.

The journal is freely available at the journal website at

$$
\text { http://www.philosophy.unimelb.edu.au/ajl/ }
$$

All issues of the journal are archived electronically at the journal website.

SUBSCRIPTIONS Individuals may subscribe to the journal by sending an email, including a full name, an institutional affiliation and an email address to the managing editor at ajl-editors@unimelb.edu.au Subscribers will receive email abstracts of accepted papers to an address of their choice. For institutional subscription, please email the managing editor at ajl-editors@unimelb.edu.au

Complete published papers may be downloaded at the journal's website at http: //www.philosophy.unimelb.edu.au/ajl/ The journal currently publishes in pdf format.

SUBMISSION The journal accepts submissions of papers electronically. To submit an article for publication, send the $\mathrm{HT}_{\mathrm{E}} \mathrm{X}$ source of a submission to a member of the editorial board. For a current list of the editorial board, consult the website.

The copyright of each article remains with the author or authors of that article. 